MacLennan, A. P. (1956). J. gen. Microbiol. 15, 485-491

\title{
The Production of Capsules, Hyaluronic Acid and Hyaluronidase by 25 Strains of Group C Streptococci
}

\author{
By A. P. MACLENNAN \\ Microbiological Research Establishment, Porton, near Salisbury, Wiltshire
}

SUMMARY: Of the 25 group $C$ streptococci examined all but 3 produced a hyaluronidase detectable in a sensitive turbidimetric test. These 3 strains and 6 of the hyaluronidase producers were heavily capsulated on serum glucose agar and in serum + glucose enriched liquid media. The capsules of all strains were readily destroyed by testicular hyaluronidase. In continually neutralized cultures of the capsulated strains hyaluronic acid was detected only transiently during logarithmic growth with the enzyme producers but rose to a high steady concentration with the other 3 strains. Hyaluronidase activity was present throughout the phase of logarithmic growth and beyond, but fell markedly in the $16 \mathrm{hr}$. following the cessation of logarithmic growth of capsulated strains in neutralized cultures and even more markedly in cultures grown without neutralization. Non-capsulated variants of the 9 capsulated organisms resembled the parent strains in enzyme production. A single passage through mice did not qualitatively influence capsulation, hyaluronic acid or hyaluronidase production by those strains examined.

McClean (1941) reported hyaluronidase production by 5 non-capsulated group C strains; 5 other strains, 4 of them capsulated, did not produce the enzyme. Crowley (1944) found that 35 of 55 non-capsulated group $\mathrm{C}$ strains of human origin produced hyaluronidase. Both these workers used the mucin clot prevention (MCP) test. More recently Di Caprio, Rantz \& Randall (1952) found that 5 group $\mathrm{C}$ strains tested produced high titres of hyaluronidase by the MCP method. Murray \& Pearce (1949), who measured enzyme activity by the degree of decapsulation of mucoid streptococci on solid media, examined 8 group C strains, only one of which produced hyaluronidase. Russell \& Sherwood (1949), using a viscosimetric method, reported hyaluronidase formation by 2 group $\mathrm{C}$ strains among 10 examined.

The production of hyaluronidase by a capsulated group $\mathrm{C}$ streptococcus in quantities too small for detection by the MCP test was described by MacLennan $(1956 a)$. It was thought worthwhile therefore to examine hyaluronidase production by other capsulated group $\mathrm{C}$ strains to see if they behaved similarly. Some non-capsulated strains were also examined by the sensitive turbidimetric test (Pike, 1948 $a$; MacLennan, 1956a) since it seemed unlikely that the methods of Murray \& Pearce (1949) and of Russell \& Sherwood (1949) were more sensitive than this test.

\section{METHODS}

Source of strains. National Collection of Type Cultures (NCTC).

Cultivation of organisms. This has been fully described elsewhere (MacLennan, $1956 a$ ). Cultures were grown from heavy inocula (saline washed) in 
serum + glucose enriched broth at $37^{\circ}$, with or without continual neutralization by sodium hydroxide.

Measurement of hyaluronic acid and hyaluronidase. Hyaluronic acid present in culture supernatant fluids was estimated by acid serum precipitation (Pike, 1946; MacLennan, 1956a). Hyaluronidase was estimated by a similar precipitation of residual hyaluronic acid after incubation of culture supernatant fluids with added polysaccharide for $16 \mathrm{hr}$. at $37^{\circ}$ at $\mathrm{pH} \mathrm{7 \cdot 2-7 \cdot 4}$ in the presence of $1 / 10,000$ thiomersalate (MacLennan, 1956a). Quinn, Seastone \& Weber (1953) reported that the measurement of hyaluronic acid by the turbidimetric method of Pike (1946) was subject to error when continually neutralized cultures were used. This, they said, was due to an increase in the buffer capacity of the cultures as neutralization proceeded, leading to a decrease in the turbidity produced by hyaluronic acid with acid serum which might be misinterpreted as being caused by hyaluronidase. It was occasionally observed here with capsulated group $\mathrm{C}$ strains in the late log phase of growth that precipitation of the hyaluronic acid in supernatants with acid serum produced finely turbid precipitates which rapidly became granular, then flocculent, and finally sedimented. These precipitates were denser to the naked eye than the normal fine stable precipitates and yet gave lower colorimeter readings. Normal turbidities could, however, be obtained by exhaustively dialysing the late log phase culture supernatant fluids against uninoculated culture medium before precipitation. Although this behaviour before dialysis leads to considerable inaccuracy in hyaluronic acid estimations it certainly does not sufficiently explain the decrease in turbidity in ageing cultures; this also occurred when these cultures were incubated with a bacteriostatic agent without further neutralization and it did not occur at low temperature (MacLennan, 1956 $a$ ) or, as will be shown, in heated cultures. The decrease in turbidity under these conditions cannot be due to an increase in buffer capacity.

Capsulation. This was best demonstrated on organisms cultivated for $24 \mathrm{hr}$. on plates of moist $20 \%$ horse serum $+1 \%$ glucose agar. UNO ink was used as a background stain (MacLennan, 1956a) as suggested by Dr Nuala Crowley (personal communication). Pl. 1 shows the even backgrounds obtained with this ink. The clear spaces in the background are due to capsular material, they are absent from smears of hyaluronidase-treated cultures.

\section{RESULTS}

All strains were plated on moist serum glucose agar following revival in blood broth from NCTC dried cultures. Sixteen strains gave non-mucoid colonies alone, the remaining 9 strains gave a mixture of mucoid and non-mucoid colonies which were separated by plating single colonies and found to breed true by serial subculture on agar. These differences in colony form were less readily obvious on fresh blood agar. On this medium all strains except 4669 were haemolytic; two strains, 4670 and 4671 , gave $\alpha$-haemolysis.

Capsulated organisms were demonstrated only in mucoid colonies, and 
suspensions of these organisms in saline were completely decapsulated by 5 min. incubation with $50 \mathrm{MCP}$ units testicular hyaluronidase $/ \mathrm{ml}$. saline suspension. Heated hyaluronidase was inactive. As others have suggested this indicates that hyaluronic acid is an essential part of these capsules. Pl. 1 shows the degrees of capsulation of the 9 capsulated strains on serum glucose agar. Rather surprisingly, perhaps, in view of the influence of anaerobiosis on the capsulation of strain 6176 in broth culture (MacLennan, 1956 a) the cultivation of all 9 capsulated strains on serum glucose agar under completely anaerobic conditions did not decrease capsulation.

Hyaluronic acid estimations were made on continually neutralized, shake cultures of the 9 capsulated strains, after the elimination of uncapsulated variants, and of two non-capsulated strains. Cultures were sampled at intervals, centrifuged, and the supernatant fluids precipitated with acid serum. Three capsulated strains produced hyaluronic acid which reached a maximum concentration in the culture supernatant fluids by the end of growth (Table 1) and was unaltered by further incubation at $37^{\circ}$ for 3 days. The cultures of the capsulated strains 6176 and 4675 , on the other hand, and of the 2 non-capsulated strains did not contain hyaluronic acid at any of the times of sampling. Although the hyaluronic acid was demonstrated in cultures of strain 6176 by more frequent sampling the rise and fall in concentration was always more rapid than that observed in earlier work (MacLennan, 1956a) with this strain. It seems likely that small differences in the composition of the medium might upset the balance of hyaluronic acid synthesis and destruction sufficiently to account for this difference in behaviour. The remaining 5 capsulated strains showed an increase in concentration of hyaluronic acid during growth, followed by a decrease similar to that previously described for strain 6176 and shown to be due to hyaluronidase action (MacLennan, 1956 $a, b$ ).

\section{Table 1. Hyaluronic acid production by group $C$ streptococci}

Culture ages (hr.)

Strain

NCTC 6178

NCTC 6176 non-mucoid

NCTC 6176 mucoid

NCTC 6177

NCTC 6180

NCTC 4675

NCTC 4676

NCTC 7912

NCTC 6963

NCTC 7022

NCTC 7023
Capsules

0

0

$+$

$+$

$+$

$+$

$+\quad 0$

$+\quad 0$

$+\quad 0$

$\begin{array}{ll}+ & \mathbf{0} \\ + & \mathbf{0}\end{array}$

$2 \overbrace{\begin{array}{llll}4 & 5 & 6 & 9\end{array}}^{24}$

Mg. hyaluronic acid/100 ml. culture

Hyaluronidase estimations on the culture supernatant fluids of the 6 capsulated enzyme-producing strains and 2 non-capsulated strains revealed enzymic activity in young cultures and throughout active growth. 
Hyaluronidase was present after $2 \mathrm{hr}$. growth although capsulation of 6 of the strains was still noted after $4 \mathrm{hr}$. The time of appearance of hyaluronidase in culture supernatant fluids was earlier and the activity apparently greater than with group A organisms (Pike, 1948b; Quinn et al. 1953, Faber \& Rosendal, 1954).

There was a distinct decrease in hyaluronidase activity in the $16 \mathrm{hr}$. following the cessation of active growth. This decrease was due, in part at least, to the thermolability of the hyaluronidase, as with strain 6176 (MacLennan, $1956 b$ ). Supernatant fluids from late logarithmic-phase cultures of the 6 capsulated hyaluronidase-producing strains were adjusted carefully to $\mathrm{pH} \mathbf{7 \cdot 2}$, heated for 1 and 2 days at $37^{\circ}$ in the presence of $1 / 10,000$ thiomersalate, then tested for hyaluronidase; there was a considerable decrease in activity. Supernatant fluids heated at $54^{\circ}$ for $2 \mathrm{hr}$. were completely inactive, further confirming the enzymic nature of the hyaluronic acid destruction.

The influence of the rapid decrease of $\mathrm{pH}$ value upon the hyaluronidase activity of unneutralized cultures containing glucose was examined. Rogers (1945) noted a suppression of hyaluronidase production in cultures of a group C streptococcus due to acid formation, and Hale (1944) showed that streptococcal hyaluronidase was rapidly inactivated at $\mathrm{pH} 4 \cdot 6$. Therefore the supernatant fluids from cultures of the 26 strains grown with neutralization until the end of logarithmic growth and also from cultures grown for $24 \mathrm{hr}$. without neutralization were tested for hyaluronidase. The $24 \mathrm{hr}$. supernatant fluids were adjusted

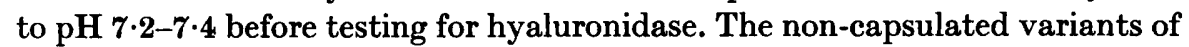
the mucoid strains and a selection of strains passaged once in mice were also tested. Table 2 summarizes the results. All non-capsulated strains, whether grown with neutralization or not, completely destroyed the added hyaluronic acid; heating the supernatant fluids at $54^{\circ}$ for $2 \mathrm{hr}$. prevented this destruction. On the other hand, although hyaluronidase-producing capsulated strains partly or completely destroyed added hyaluronic acid when neutralized cultures were used, the supernatant fluids from $24 \mathrm{hr}$. unneutralized cultures showed little or no activity. Since this was true also of non-capsulated variants of these 9 strains, there was no suggestion that the absence of capsulation was due to increased hyaluronidase synthesis, in agreement with previous work with strain 6176 (MacLennan, 1956a).

A single mouse passage of a number of strains by the intraperitoneal route causing death within $24 \mathrm{hr}$. did not qualitatively influence the production of capsules, hyaluronic acid or hyaluronidase (Table 2).

\section{DISCUSSION}

By a sensitive test more group $\mathrm{C}$ streptococci have now been shown to produce hyaluronidase than has previously been reported. In addition to using a sensitive test, early sampling and continual neutralization are important for the demonstration of hyaluronidase production by capsulated organisms and their non-capsulated variants but not for that produced by non-capsulated group $\mathrm{C}$ strains. The time of appearance of the enzyme in cultures of capsulated 


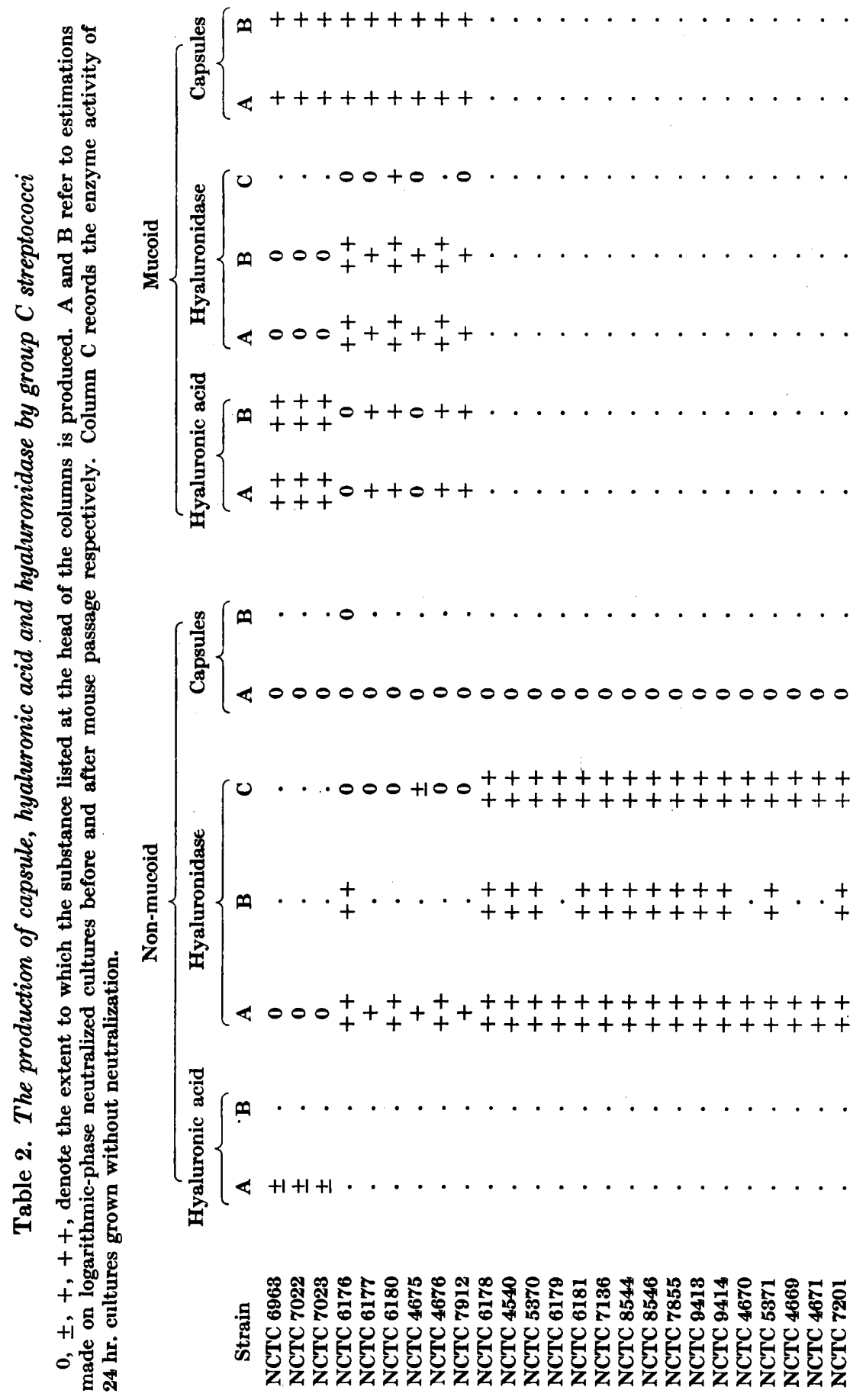


strains is earlier and the activity apparently greater than with group $\mathbf{A}$ organisms (Pike, 1948b; Faber \& Rosendal, 1954), although differences in culture media and size of inoculum may partly account for this.

With capsulated strains which produce hyaluronidase, hyaluronic acid was either detected only over a short period of culture growth or not at all. This is probably due more to a rapid destruction of hyaluronic acid by hyaluronidase than to gross variation in the amount of hyaluronic acid synthesized, since some of these strains are as well capsulated on solid media as three strains which do not produce hyaluronidase and liberate large amounts of hyaluronic acid in broth culture.

The appearance of capsules on solid or liquid media can be completely suppressed by cultivation in the presence of added hyaluronidase (McClean, 1941 ; Murray \& Pearce, 1949); it is therefore possible that some non-capsulated, hyaluronidase-producing streptococci also produce hyaluronic acid but that, because of the production of more hyaluronidase than in capsulated strains, a capsule is never formed, even on solid media. The report that a penicillinresistant variant of a non-mucoid hyaluronidase-producing group A streptococcus (Faber \& Rosendal, 1955) produced hyaluronic acid supports this idea, particularly as there was an inhibition of release of hyaluronidase from this variant organism during the exponential growth phase. On the other hand, since the non-capsulated variants of 3 capsulated strains resemble their parents in not producing hyaluronidase and since there is no detectable increase in enzyme production by non-capsulated variants of capsulated, hyaluronidase-producing strains (Table 2), the absence of hyaluronic acid and capsules from some strains is clearly due to inability to synthesize the polysaccharide.

I wish to thank Dr D. McClean for his interest in this work, DrI. A. Macpherson for taking photographs and Mr D. C. Hawkins for technical assistance.

\section{REFERENCES}

Crowley, N. (1944). Hyaluronidase production by haemolytic streptococci of human origin. J. Path. Bact. 56, 27.

Di Caprio, J. M., Rantz, L. A. \& Randall, E. (1952). Studies on streptococcal hyaluronidase and antihyaluronidase. Arch. inter. Med. 89, 374.

Faber, V. \& Rosendal, K. (1954). Streptococcal hyaluronidase. II. Studies on the production of hyaluronic acid and hyaluronidase by representatives of all types of haemolytic streptococci belonging to group A. Acta path. microbiol. scand. 35, 159.

Faber, V. \& Rosendal, K. (1955). Streptococcal hyaluronidase. IV. The effect of penicillin on the production of hyaluronic acid and hyaluronidase by haemolytic streptococci (type 4, group A). Acta path. microbiol. scand. 37, 286.

Hale, C. W. (1944). Studies on diffusing factors. 5. The influence of some environmental conditions on the activity of hyaluronidase. Biochem. $J .38,368$.

MCClean, D. (1941). The capsulation of streptococci and its relation to diffusion factor (hyaluronidase). J. Path. Bact. 53, 13.

Maclennan, A. P. (1956a). The production of capsules, hyaluronic acid and hyaluronidase by group $\mathrm{A}$ and group $\mathrm{C}$ streptococci. J. gen. Microbiol. 14, 134. 


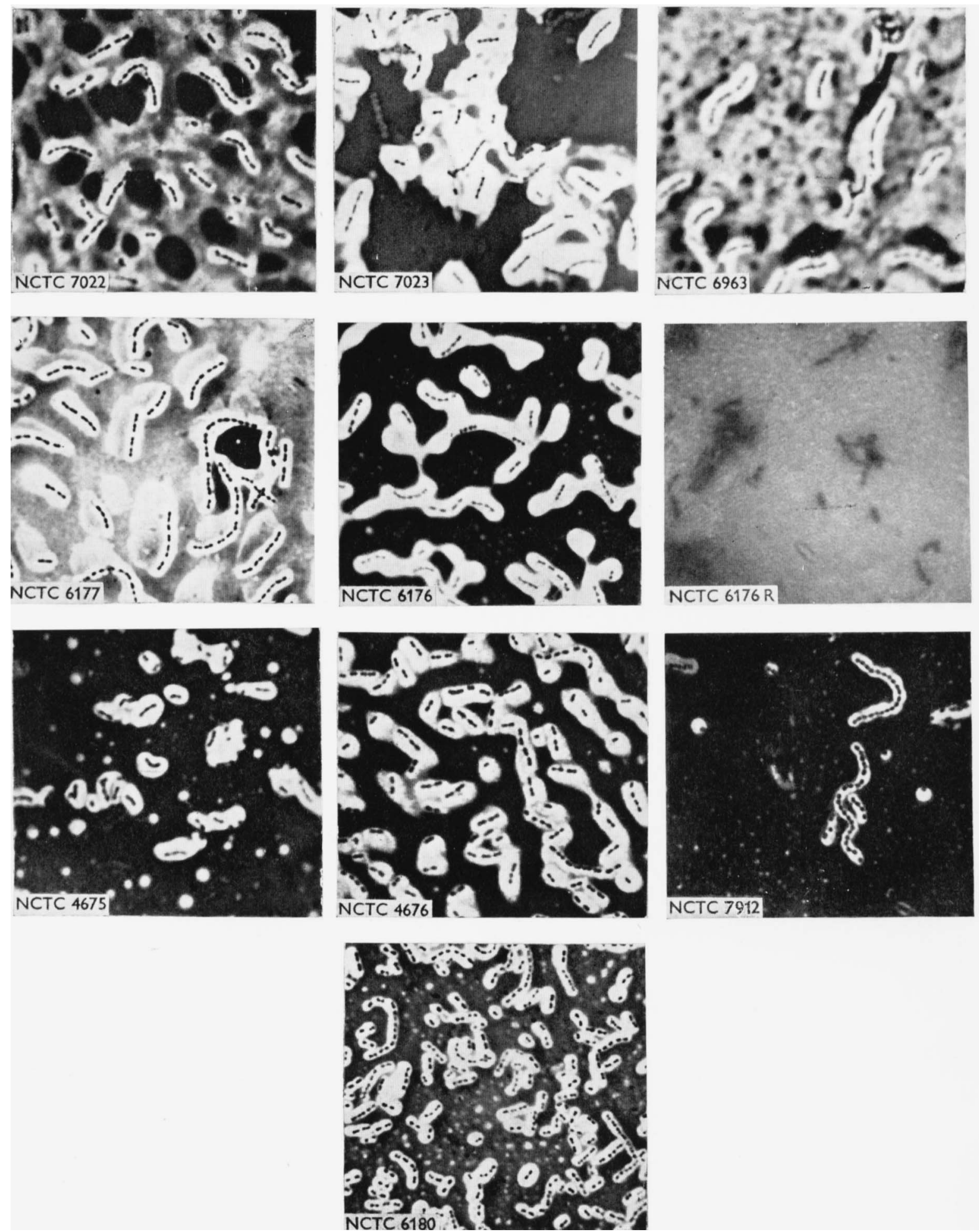

A. P. Maclennan-Production of capsules, myaluronic acil ani) HYALURONIDASE BY 25 STRAINS OF GROUP C STREPTOCOCCI 
Maclennan, A. P. (1956 b). The isolation and characterization of a hyaluronidase produced by a capsulated strain of group $\mathrm{C}$ streptococcus. J. gen. Microbiol. 14, 143.

Murray, R. G. E. \& Pearce, R. H. (1949). The detection and assay of hyaluronidase by means of mucoid streptococci. Canad. J. Res. E, 27, 254.

Pike, R. M. (1946). A study of group A streptococci from healthy carriers with particular reference to mucoid polysaccharide production. J. infect. Dis. 79, 148.

Pike, R. M. (1948 $a$ ). Streptococcal hyaluronic acid and hyaluronidase. I. Hyaluronidase activity of non-capsulated group A streptococci. J. infect. Dis. 83, 1.

Pike, R. M. (1948b). Streptococcal hyaluronic acid and hyaluronidase. II. Production and subsequent destruction of hyaluronic acid by certain strains of group A streptococci. J. infect. Dis. 83, 12.

Quinn, R. W., Seastone, C. V. \& Weber, R. W. (1953). The relationship of the antigenic characteristics of streptococci and specific antibody responses following streptococcal infections. $J$. infect. Dis. 93, 57.

Rogers, H. J. (1945). The conditions controlling the production of hyaluronidase by micro-organisms grown in simplified media. Biochem. J. 39, 435.

Russfit, B. E. \& Sherwood, N. P. (1949). Studies on streptococci. II. The role of hyaluronidase in experimental streptococcal infection. J. infect. Dis. 84, 81.

\section{EXPLANATION OF PLATE}

Capsule production by group $C$ streptococci. All strains except 7022, 7023 and 6963 produce hyaluronidase. $6176 \mathrm{R}$ is a non-capsulated variant of 6176. All strains were grown on $20 \%(v / v)$ horse serum $+1 \%(w / v)$ glucose nutrient agar for $24 \mathrm{hr}$. 